\title{
An Investigation of "San Carlos Olivine": Comparing USNM-distributed Material with Commercially Available Material
}

\author{
John H. Fournelle*
}

* Eugene Cameron EPMA Lab, Dept. of Geoscience, University of Wisconsin, Madison, WI 53706

Natural Forsterite-rich olivine [1], of mantle xenolith origin, is a standard for $\mathrm{Mg}$ and $\mathrm{Si}$ in many geoscience electron microprobe labs. Many labs use the San Carlos Fo90.1 standard developed by Gene Jarosewich and co-workers (1980) at the Smithsonian: USNM 111312/444 ]2].

In the normal course of operation of the UW-Madison EPMA lab in 2008-9, non-USNM-distributed crystals of San Carlos olivine were encountered, being utilized in scientific research (for SIMS oxygen isotope standards, and for simulation of lunar solar wind bombardment). EPMA of these olivines showed consistent differences from the USNM-distributed olivine: one set was more Fe-rich (Fo89.2) and the other, more Mg-rich (F91.1). This led to further detailed investigation of USNM San Carlos olivine and the range of compositions of those grains potentially used by EPMA labs for standards.

Larger crystals of "San Carlos olivine" are available commercially. Three 1-2 mm crystals from one source (SIMS oxygen standards) were analyzed by EPMA for $\mathrm{Si}, \mathrm{Mg}$ and $\mathrm{Fe}$, and a consistent value of Fo89.2 ( \pm 0.2$)$ was found. Thirty-six $1 \mathrm{~cm}$-size crystals from another source were analyzed (1566 spot analyses; 30-40 points per crystal) and a range of compositions from Fo90.5 to Fo91.4 was found (average Fo91.1 \pm 0.3 ). The compositional range (in elemental weight percentage) is shown in Table 1 and a histrogram of observed values is shown in Figure 1. Having found this variability, a study was then done to determine the range of variability possible in the USNM San Carlos olivine standard.

Amelia Logan of the Smithsonian supplied 2 small vials of the USNM111312/44 material. Jarosewich et al (1980) had calculated "Boyd homogeneity indices" for USNM standards, with 100 measurements on 10 grains of each standard, and values $<3$ were considered OK. I report 71 measurements on 23 small (200-300um) grains of USNM 111312/44 in 1 vial, which show a bit wider range of heterogeneity in $\mathrm{Si}$ and $\mathrm{Mg}$ than originally found in the 1980 paper: $\mathrm{Si} 2.32$ (vs 0.81 ), Mg 3.05 (vs 1.00), and Fe 1.2 (vs 0.9). Data are shown in Figure 2 histogram. These "Boyd" numbers are less easy to comprehend than a simple k-ratio criteria: take the average x-ray count of a large number of points (which will represent the wet chemical analysis), and then take the lowest and the highest counts, to represent the worst case scenario. Here the results were: Si ranged from 0.947 to 1.017 (5\% max difference), $\mathrm{Mg} 0.969$ to 1.043 (4\% max) and $\mathrm{Fe} 0.946$ to 1.055 (6\% max). The good news is that $82 \%$ of the analyses fell within the Fo90.1 ( \pm 0.2$)$ window.

Conclusions: (1) Crystals of "San Carlos olivine" available from mineral dealers cannot be assumed to be of the same composition as USNM 111312/444. (2) There is a small but not unreasonable probability that EPMA users who assume that any ONE grain of USNM Carlos olivine is EXACTLY the published composition could be making an error of $\sim 5 \%$ in the measured K-ratio for $\mathrm{Mg}$, Si or Fe. EPMAers need to heed the solid advice of Gene Jarosewich, to acquire "a reasonably large number of counts on a reasonably large number of grains" (Jarosewich et al, 1980). (3) It is a 
beneficial operating procedure for an EPMA lab to run several standards for each element where possible, and then compare the results for consistency. (4) Periodic use of applications such as "Evaluate" (Probe for EPMA software) provides one way to cross-check all standards and determine whether some grains of well known standards may not be exactly the published values and either should not be used, or should have their stated compositions modified.

QC Proposal: That a probe mount of at least 25 grains of USNM 111312/444 San Carlos olivine be made available to any EPMA lab for a short period of time, to run as a primary standard, to compare one's own few grains with and decide whether or not those grains' composition are of the exact composition being used, or whether small modifications might be justified.

\section{References}

[1] Forsterite (abbreviated Fo) is the $\mathrm{Mg} 2 \mathrm{SiO} 4$ component of olivine; Fo numbers are atomic \%.

[2] Jarosewich et al, Geostandards Newsletter 4 (1980), 43; errata, 4 (1980) 257.

\section{TABLE 1. Range of olivine compositions}

\begin{tabular}{llll}
\hline Fo\# & Mg wt $\%$ & Fe wt $\%$ & Si wt $\%$ \\
\hline 87 & 28.41 & 9.75 & 18.86 \\
88 & 28.85 & 9.04 & 18.94 \\
89 & 29.31 & 8.32 & 19.02 \\
90 & 29.76 & 7.60 & 19.11 \\
91 & 30.22 & 6.87 & 19.19 \\
92 & 30.69 & 613 & 19.27 \\
\hline
\end{tabular}
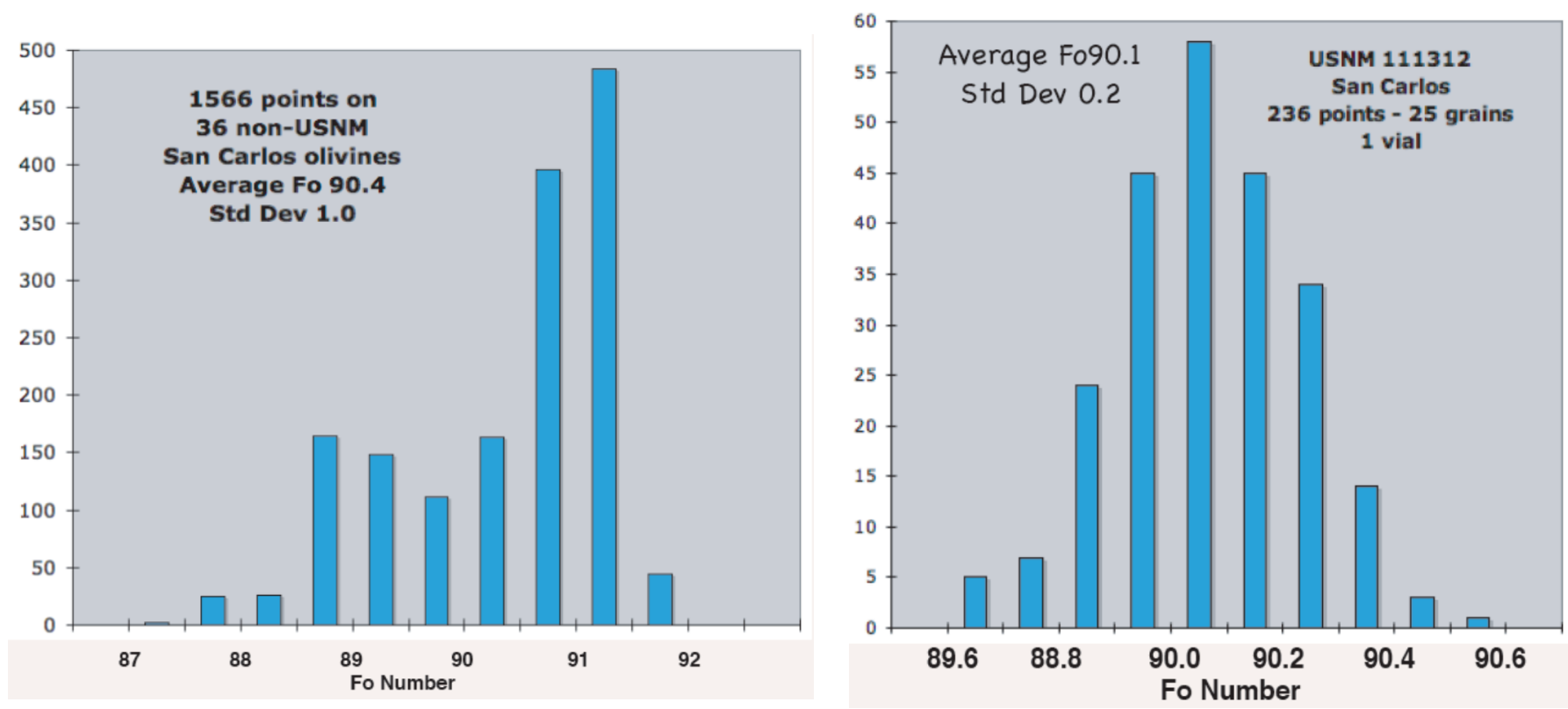

Figure 1

Figure 2 